\title{
Hemophagocytic Syndrome
}

\author{
Manjusha Shripad Dhawale • Anil R. Joshi • \\ Milind Anil Bhatkule • Neela Ratnakar Kumbhakarna • \\ Rajan Shamrao Bindu
}

Received: 30 April 2011/Accepted: 22 November 2012/Published online: 18 December 2012

(C) Indian Society of Haematology \& Transfusion Medicine 2012

\begin{abstract}
Hemophagocytic syndrome is a life threatening condition characterized by uncontrolled hyperinflammation on the basis of various inherited or acquired immune deficiencies. We report a case of hemophagocytic syndrome in a 18 years old male with a brief review of literature.
\end{abstract}

Keywords Hemophagocytic syndrome . Immunodeficiency

\section{Introduction}

Dysregulation of immune system can sometimes cause cytokine-mediated inappropriate stimulation and proliferation of macrophages, which subsequently phagocytose self blood cells, causing a distinct clinicopathologic entity called hemophagocytic syndrome or hemophagocytic lymphohistiocytosis [1-3]. There is benign proliferation of the mature macrophages and uncontrolled phagocytosis of the platelets, erythrocytes, lymphocytes and their hematopoietic precursors in the bone marrow and other reticuloendothelial organs, giving rise to the cytopenias [2, 3]. Clinically fever, splenomegaly, lymphadenopathy, jaundice, rash, elevated triglyceride and ferritin levels and low fibrinogen level characterize this systemic disorder [2-5]. Two forms, primary (familial) and secondary (acquired) are recognized $[4,6,7]$. The Histiocytic Society Protocol HLH2004 allows diagnosis of hemophagocytic syndrome after

M. S. Dhawale · A. R. Joshi · M. A. Bhatkule $(\bowtie) \cdot$

N. R. Kumbhakarna $\cdot$ R. S. Bindu

Department of Pathology, Government Medical College and

Hospital, C/O L.C. Deo 39, SBH Colony, Near Peer Bazaar,

Osmanpura, Aurangabad 431003, Maharashtra, India

e-mail: drmilindbhatkule@yahoo.co.in meeting at least five out of its eight criteria of fever ( $\geq 7$ days), splenomegaly, cytopenia [ $\geq 2$ lineages; anemia ( $\mathrm{Hb}<9 \mathrm{~g} / \mathrm{dL})$, thrombocytopenia $(<1 \mathrm{lac} / \mathrm{cmm})$, neutropenia $(<1,000 / \mathrm{cmm})]$, hypertriglyceridemia $(\geq 265 \mathrm{mg} / \mathrm{dL})$ and/or hypofibrinogenemia $(<1.5 \mathrm{~g} / \mathrm{L})$, hemophagocytosis (bone marrow, spleen, lymph nodes), low/absent natural killer cell activity, hyperferritinemia $(\geq 500 \mathrm{mcg} / \mathrm{L})$, increased soluble CD25 $>2,400$ units/mL $[2,8]$.

\section{Case Report}

An 18 years old male patient presented with 2 months' history of fever, generalised weakness and weight loss. His general condition had been deteriorating progressively. Physical examination revealed gross pallor, wasting, palpable spleen $8 \mathrm{~cm}$ below the left costal arch. There was no palpable lymphadenopathy or rash. The complete blood count revealed a pancytopenia like picture with hemoglobin $6 \mathrm{~g} / \mathrm{dL}$, total leucocyte count $4,300 / \mathrm{cmm}$, platelet $90,000 /$ $\mathrm{cmm}$. His peripheral smear was negative for malarial parasite. Bone marrow aspiration was performed which revealed hypocellularity with a normal myeloid-erythroid ratio and normal maturation. Conspicuously, there was an increased number of macrophages, most of which contained engulfed RBC, neutrophils, normoblasts, platelets and nuclear debris (Figs. 1, 2). All the above findings raised a strong suspicion of hemophagocytic syndrome which was affirmatively confirmed by the raised levels of serum ferritin $(2,221.55 \mathrm{mcg} / \mathrm{L})$, serum triglycerides $(251 \mathrm{mg} / \mathrm{dL})$, serum LDH (850 IU/L) and decreased fibrinogen level $(1.3 \mathrm{~g} / \mathrm{L})$.

After fulfilling six out of eight criteria of HLH-2004, the patient was diagnosed as a case of hemophagocytic syndrome/hemophagocytic lymphohistiocytosis.

While an imaging and serological work-up for any underlying bacterial, fungal or viral infection or any 


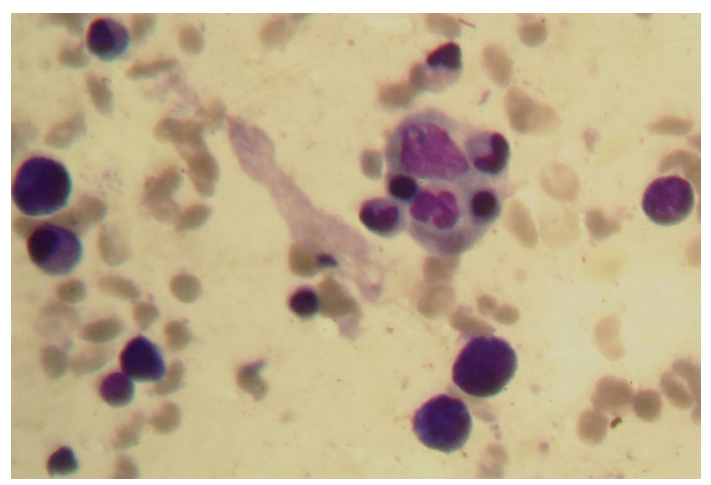

Fig. 1 Increased number of macrophages engulfing the normoblasts and neutrophil in bone marrow

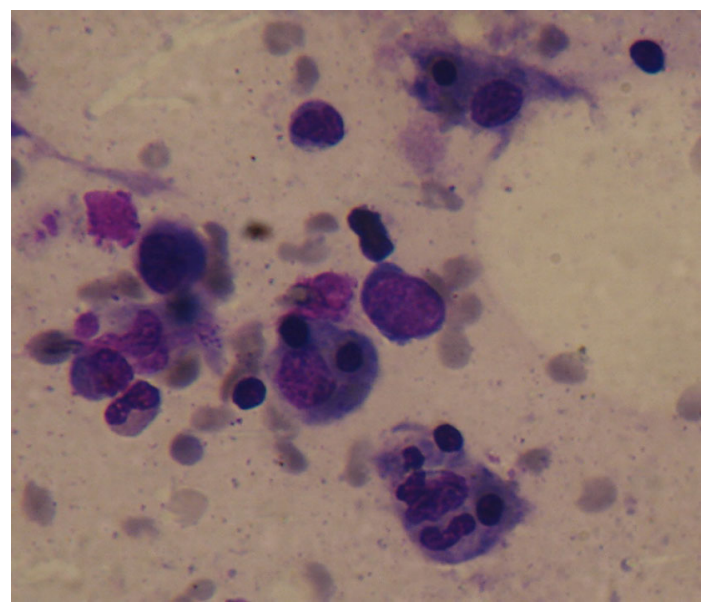

Fig. 2 Macrophsges engulfing the lymphocytes, normoblast and neutrophil in bone marrow

hematolymphoid or other malignancy was started, the patient was prescribed empirical low dose steroid therapy to which he responded favourably. His general condition improved, fever resolved and blood counts started improving. He still had bilateral pleural effusion. His pleural fluid was sent for cytology which revealed the presence of lymphocytes and was negative for acid fast bacilli. Radioimaging findings failed to reveal the presence of any tubercular or malignant focus. Finally, the sample of pleural fluid sent for M. tuberculosis PCR was reported positive and the patient has since been on DOTS regimen and has responded extremely well.

\section{Discussion}

HPS is a life threatening condition characterized by uncontrollable hyperinflammation due to cytokine-mediated dysregulated macrophage activation and proliferation. Familial HPS is an autosomal recessive disorder, seen in 1 in 50,000 births [4]. Usually presenting in infancy, it is uniformly fatal if untreated and allogenic bone marrow transplantation is the therapy of choice $[4,6]$. Acquired HPS is associated with various stimuli-like infections (EBV most common; also fungal, mycobacterial, parasitic), hematolymphoid and other malignancies, drugs and immunocompromised states [1-6]. The index case had an underlying tubercular infection and therefore, was a case of secondary or acquired HPS. The outcome varies but treatment of underlying infection can result in rapid resolution, as seen in our patient. Whatever the underlying cause might be, it is postulated that elaboration of activating cytokines viz. interferon- $\gamma$, tumor necrosis factor (TNF)- $\alpha$, interleukins-1, 2 and 6 by T-helper cells promotes activation of macrophages in this disease [2, 6]. Phagocytosis of blood cells and their precursors seen in bone marrow, spleen, and lymph nodes is a hallmark feature. Activated macrophages may engulf erythrocytes, leukocytes, and platelets, their precursors, and cellular fragments. These cells appear "stuffed" with other blood cells [6]. Because so many immunologic, neoplastic, genetic, and infectious disorders may be associated with HPS, clinicians should work closely with pathologists and microbiologists to clearly define precipitating or underlying illnesses. The aim of therapy is suppression of the increased inflammatory response by immunosuppressive/ immunomodulatory agents, cytotoxic drugs $[1,2,4,8]$. Moreover, treatment of the underlying infection can result in rapid resolution of the disease. As genetic cases can only be cured with stem cell transplantation, genetic testing is advisable in diagnosed patients to recognize familial cases $[5,6,8]$. If a patient presents with fever and unexplained cytopenias, it is important to consider hemophagocytic syndrome as a potential diagnosis which calls for a thorough work-up to rule out any infection or malignancy. Awareness and high vigilance on the part of the clinician and the pathologist can save many a precious lives especially those with an underlying cause amenable to therapy.

\section{References}

1. Kumakura S (2005) Hemophagocytic syndrome. Intern Med 44(4):278-280

2. Gupta AP, Parate SN, Bobhate SK, Anupriya (2009) Hemophagocytic syndrome: a cause for fatal outcome in tuberculosis. Indian J Pathol Microbiol 52(2):260-262

3. Raka S, Nayar P, Godbole R, Manchanda R (2009) Familial hemophagocytic lymphohistiocytosis. Indian J Hematol Blood Transfus 25(2):78-80

4. Sadek SA, Terkait NA (2008) Difficulties in the diagnosis of familial hemophagocytic lymphohistiocytosis. Kuwait Med J 40(3):241-243

5. Das S, Kalyani R (2008) Hemophagocytic syndrome. Indian J Pathol Microbiol 51(1):125-126 
6. Fisman DN (2000) Hemophagocytic syndromes and infection. Emerg Infect Dis 6(6):601-608

7. Fahmi YK, Fawzy Z, Siddiqui I, Yassin MA (2006) Hemophagocytosis and military tuberculosis in a patient in the intensive care unit. Indian J Crit Care Med 10(2):112-114
8. Henter JI, Horne A, Arico M, Egeler RM, Filipovich AH, Imashuku S et al (2007) HLH-2004: diagnostic and therapeutic guidelines for hemophagocytic lymphohistiocytosis. Pediatr Blood Cancer 48:124-131 